Proc. Indian Acad. Sci., Vol. 88 A, Part II, Number 1, March 1979, pp. 41-49, $\Theta$ printed in India.

\title{
On the interpretation of velocities derived from spaced fading records
}

\author{
H CHANDRA \\ Department of Physics, University of Adelaide, South Australia 5001 \\ Present address: Physical Research Laboratory, Ahmedabad 380009 \\ MS received 22 February 1978; revised 22 September 1978
}

\begin{abstract}
A number of spaced fading records obtained at Buckland Park, South Australia, using partial reflection technique (at $80-100 \mathrm{~km}$ ) have been subjected to different methods of analysis, viz., time delay methods, the full correlation method and the cross-spectrum method. True drift velocities derived from the full correlation method appear to be the most correct. The variation of velocity with fading frequency obtained from cross-spectrum method is most likely to be due to the variation of velocity with time within the record itself.
\end{abstract}

Keywords. Partial reflection drift; ionospheric drift; spaced receiver method.

\section{Introduction}

Considerable interest has been shown recently in the spaced receiver method of measuring drift motion of ionisation irregularities in the ionosphere. This revival has been possible due to (i) experiments successfully testing the basic assumptions of the method by employing a large array of aerials (such as at Eucklard Fark in South Australia by Felgate 1970 and by Golley and Rossitor 1971), (ii) improvements in the instrumentation which has enabled digital recording of the data at fixed ionospheric heights, e.g., using partial reflection from $D$-region, or using multjfrequency system (Kinosonde) for use in $E$ or $F$ region, and (iii) successful comparison at different levels of the ionosphere between the velocities derived from spaced receiver technique and those simultaneously derived from other independent methods, i.e., meteor winds (Stubbs 1973; Felgate et al 1975; Wright et al 1976a), rocket methods (Vincent et al 1977), incoherent scatter method (Wright et al 1976b) and back-scatter radar (at equator, Crochet et al 1977).

Different methods of reducing spaced fading records have been followed by different workers and in the absence of any standard recommended method intercomparison of results at different locations may become difficult. To arrive at some standard method of analysis, it is essential to inter-compare different methods of analysis that are commonly used. With this aim in view certain $D$ region fading records $(80-100 \mathrm{~km})$ at Buckland Park have been subjected to different methods of analysis. 


\section{Common methods of analysis}

\subsection{Similar fade method}

This is the simplest method of reducing spaced fading records and is also known as Mitra's method (Mitra 1949). Individual fades are identified and time delays between different pairs of aerials measured and from the mean time delays one can easily compute drift velocity. This velocity is referred to as the apparent drift velocity $\left(V_{a}\right)$. Sprenger and Schminder (1969) suggested another variant of this method where velocities are derived from individual time delays and later averaged. This is known as variant ${ }_{2}$ method whereas the original Mitra's method is known as variant 1 method.

\subsection{Full correlation method}

Mitra (1949) had assumed that the diffraction pattern at ground does not deform as it moves. Briggs et al (1950) developed a correlation method of analysis, where one can determine the steady drift velocity of the pattern, known as the true drift velocity $(V)$, as well as a measure of the changes in the pattern in terms of a parameter known as characteristic velocity $\left(V_{0}\right)$. The apparent drift speed and the true drift speed are related by the equation:

$$
V_{\mathrm{a}}=V\left[1+\left(V_{\mathrm{o}} / V\right)^{2}\right] \text {. }
$$

Phillips and Spencer (1955) extended this method to anisometric patterns while Fooks (1965) gave it a computerised mathematical form. Sprenger and Schminder

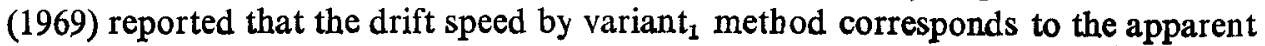
drift speed $V_{a}$ while the drift speed by variant 2 method corresponds to the true drift speed $V$.

One of the basic assumptions in the application of correlation analyses, i.e., the temporal and spatial correlation functions are similar, has been tested using the large antenna array at Buckland Park where drift velocity derived by spatial correlation analyses was found to agree with the drift velocity derived by normal temporal correlation analyses. The meaning of the characteristic velocity $V_{0}$ has been tested by the computer simulation work (Pitteway et al 1971).

\subsection{Cross spectrum method}

A different approach is to Fourier-analyse the amplitude data and then estimate time delays at different Fourier frequencies present in the fading pattern (Jones and Maude 1965). This method determines only the apparent drift velocity at different Fourier frequencies and in the presence of wave motion in the ionosphere will show a variation of the drift velocity with frequency. One has to be cautious, however, as one may get such variations due to other reasons also like variation of velocity with time (Felgate and Golley 1971) or presence of different scale sizes. Most of the authors have reported either an increase in the velocity with frequency or no systematic variation (Briggs 1977). 


\subsection{Full correlation method with filtering the fading records}

Sprenger and Schminder (1969) adopted another method of studying the variation of velocity with frequency. The amplitude data were subjected to various high pass filters and correlation analysis performed each time. True drift velocity increased with filter frequency while the apparent drift velocity remained independent of filter frequency. Chandra and Briggs (1978) observed similar results with high pass filters from the $D$-region records at Buckland Park. They also observed decrease of the true drift velocity when low pass filters are used. These variations in the true drift velocity have been shown to be inevitable when correlation analysis is used and should not be considered as an evidence for dispersive motion (Chandra and Briggs 1978).

\section{Comparison of Variant Vand $_{1}$ Variant 2 velocities}

About fifteen records of 3-min extent (450 digitised samples) were subjected to time delay method analyses. For this the digital data were computer-plotted on a magnified time scale and the individual time delays measured manually. To find a quick solution of applying such analysis on a digital form of data cross-correlograms were computed for every 10 -sec of data ( 45 samples) and time delays measured by fitting a Gaussian near the peak (as is done in the normal correlation analysis). From about eighteen sets of time delays thus obtained both variant $t_{1}$ and variant $t_{2}$ methods were used and the velocities obtained $(55 \mathrm{~m} / \mathrm{s}$ and $41 \mathrm{~m} / \mathrm{s}$ for variant

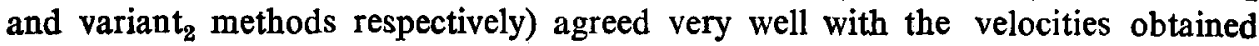
in the manual similar fade method $(56 \mathrm{~m} / \mathrm{s}$ and $45 \mathrm{~m} / \mathrm{s})$.

Comparison of speeds obtained from time delay methods with those obtained from full correlation analyses are shown in figures 1a and 1b. The apparent drift speed computed from the optimum cross correlation $\left(V_{a}\right)$ agrees well with the variant $_{1}$ method and a correlation of 0.83 is obtained between these two. The true drift speed $V$ from correlation analysis is however smaller than that with the variant $_{2}$ method and a correlation of only 0.37 is obtained in this comparison. A comparison of the ratio $V_{a} / V$ with that of the ratio $V^{\prime}$ variant $_{1} / V^{\prime}$ variant shows $_{2}$ that there exists a reasonably good agreement between the two as long as $V_{a} / V$ is less than about $1 \cdot 5$.

\section{Meaning of apparent and true drift speeds}

To understand the relative merits of the apparent and true drift speeds a continuous record of a sufficient duration was analysed by full correlation analysis at different sample length intervals and in figure 2 a continuous $30 \mathrm{~min}$ computations are plotted. In the upper portion results from $3 \mathrm{~min}$ sample length are presented while in the lower portion results from $1 \mathrm{~min}$ of sample are presented. From these variations, it is evident that the true drift speed gives a more consistent picture while at times there may be very odd value of apparent drift speed (for example $V_{a}$ at $1458 \mathrm{hr}$ ). Thus very high values of apparent drift speed must be rejected as these may arise when there are sudden changes in the direction of time shift within the length of the record (as seen from the amplitude fading records of this sample). 

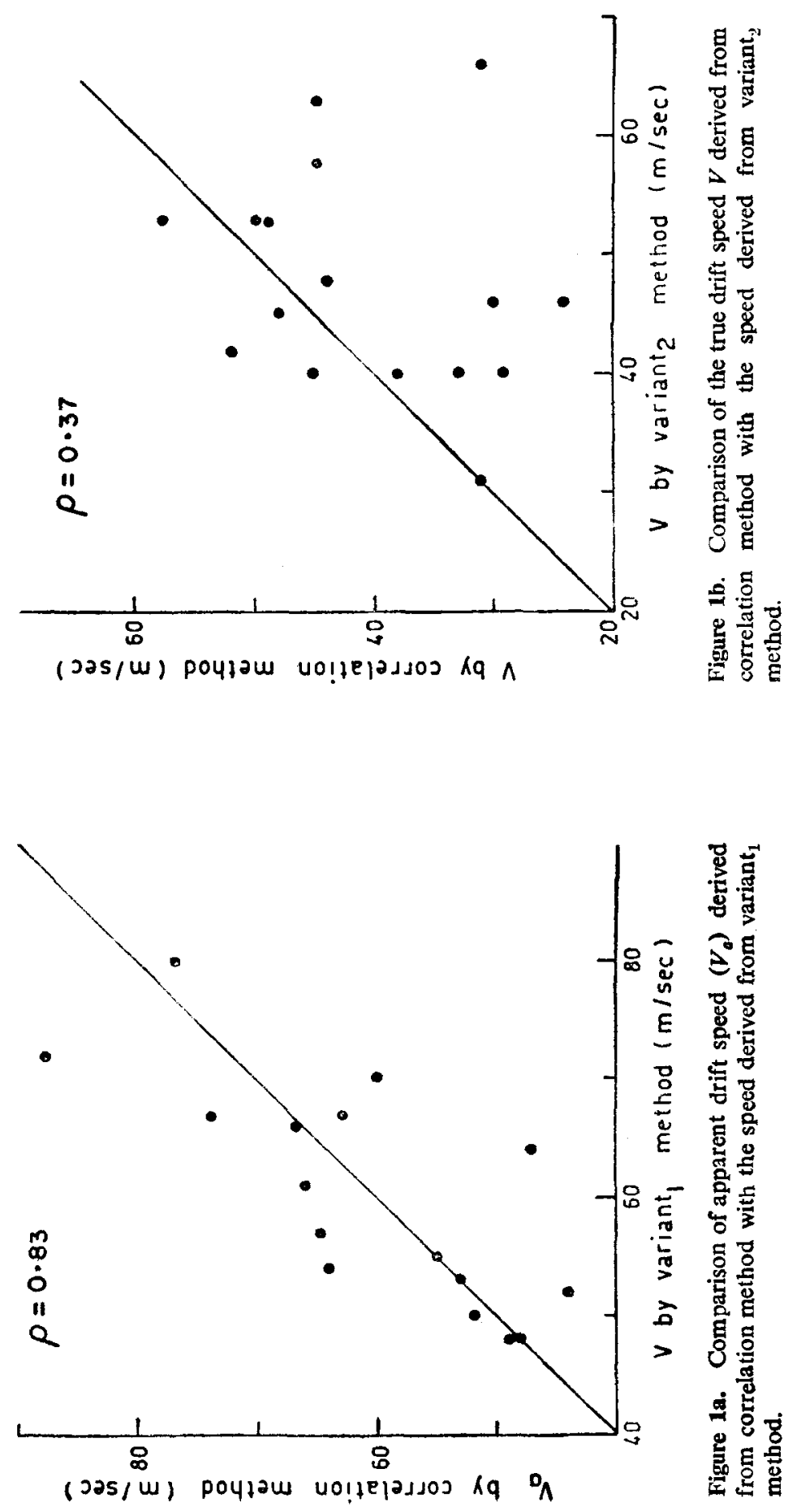


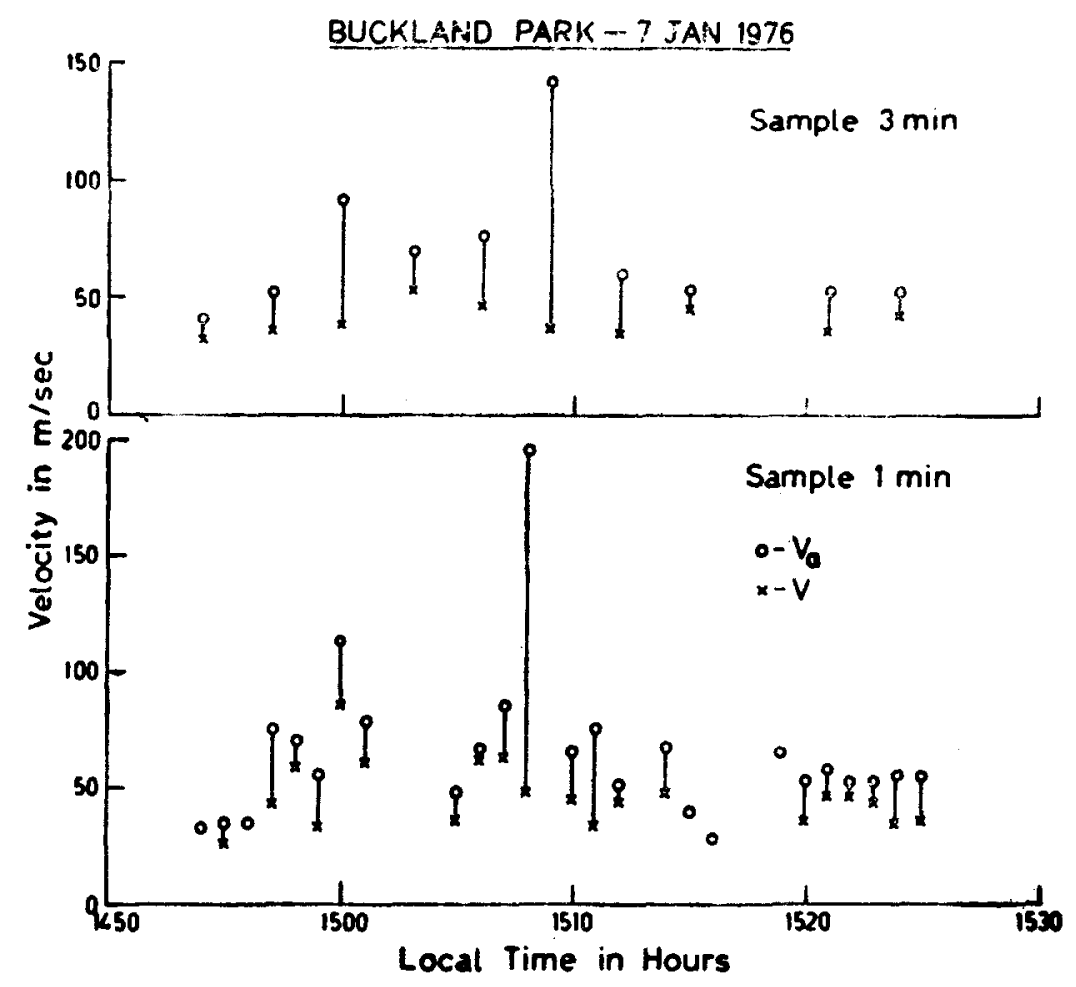

Figure 2. Variation of $V_{a}$ and $V$ derived from correlation methods using sample lengths of $1 \mathrm{~min}$ and $3 \mathrm{~min}$.

\section{Drift speed from different aerial configurations}

The amplitude recording both at Buckland Park and at Woomera are done on 4 antennas simultaneously and one set or sometimes more than one set of antenna configurations are used in the data analyses. In figure 3 one such comparison is made when drift speeds are computed from two different right angled triangles of identical separations. The open circles denote the true drift speeds obtained from one such set while crosses denote the true drift speeds from another triangle. Both the zonal (E-W) and the meridional (N-S) components of drift speed match well at different hours on this day. The comparison at the height $87 \mathrm{~km}$ being better than the comparison at $91 \mathrm{~km}$.

\section{Results of dispersion analysis}

A large number of records were subjected to the cross-spectrum aralysis to find out apparent drift velocities at different Fourier components. W hile a number of records showed a positive dispersion there were many records where no systematic variation of the velocity with frequency could be obtaired. A few cases 


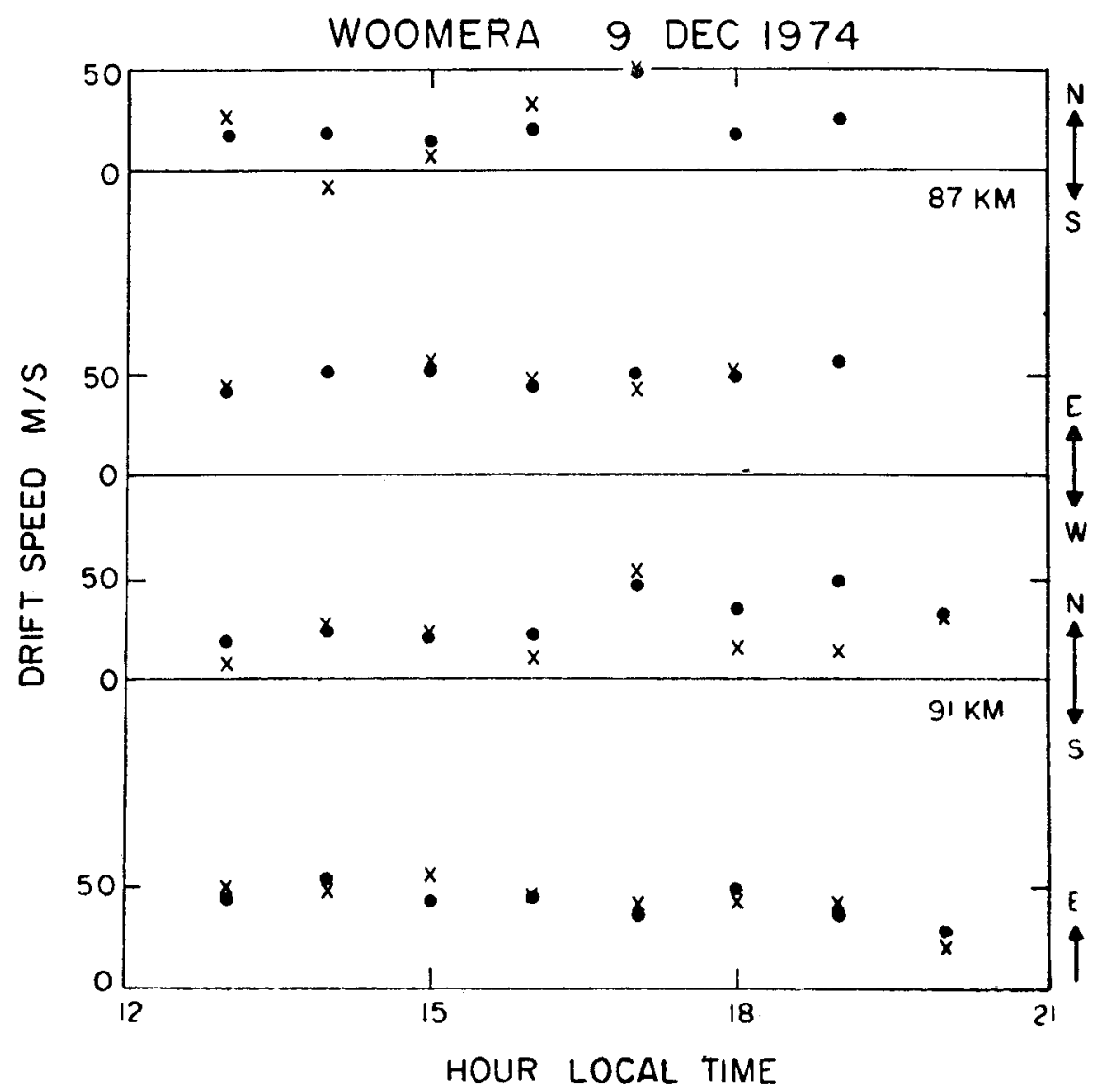

Figure 3. Comparison of the drift components along N-S and E-W axes derived from correlation method using two different isosceles right-angled triangular configurations of identical dimensions.

(three) when positive dispersion in drift speed was obtained are plotted in figure 4. It must be noted that the amount of scatter in the record is roughly proportional to the ratio $V_{a} / V$. Larger the ratio $V_{a} / V$, larger is the variation in the speed. As large ratio of $V_{\mathrm{a}} / V$ is normally for records, where there is a variation of velocity with time within the record, it implies that a large positive dispersion could just be present due to variation in velocity with time within the record rather than due to the wave motion.

\section{Results with filtering of data}

A large number of records were subjected to various low pass and high pass filters before full correlation analysis was performed and the average variation of the true drift speed $V$ and the apparent drift speed $V_{a}$ with filter frequency in both cases are shown in figure 5. Records of 6 min sample length were used in this type of analysis so that after filtering the central $3 \mathrm{~min}$ portion of the record, could be 


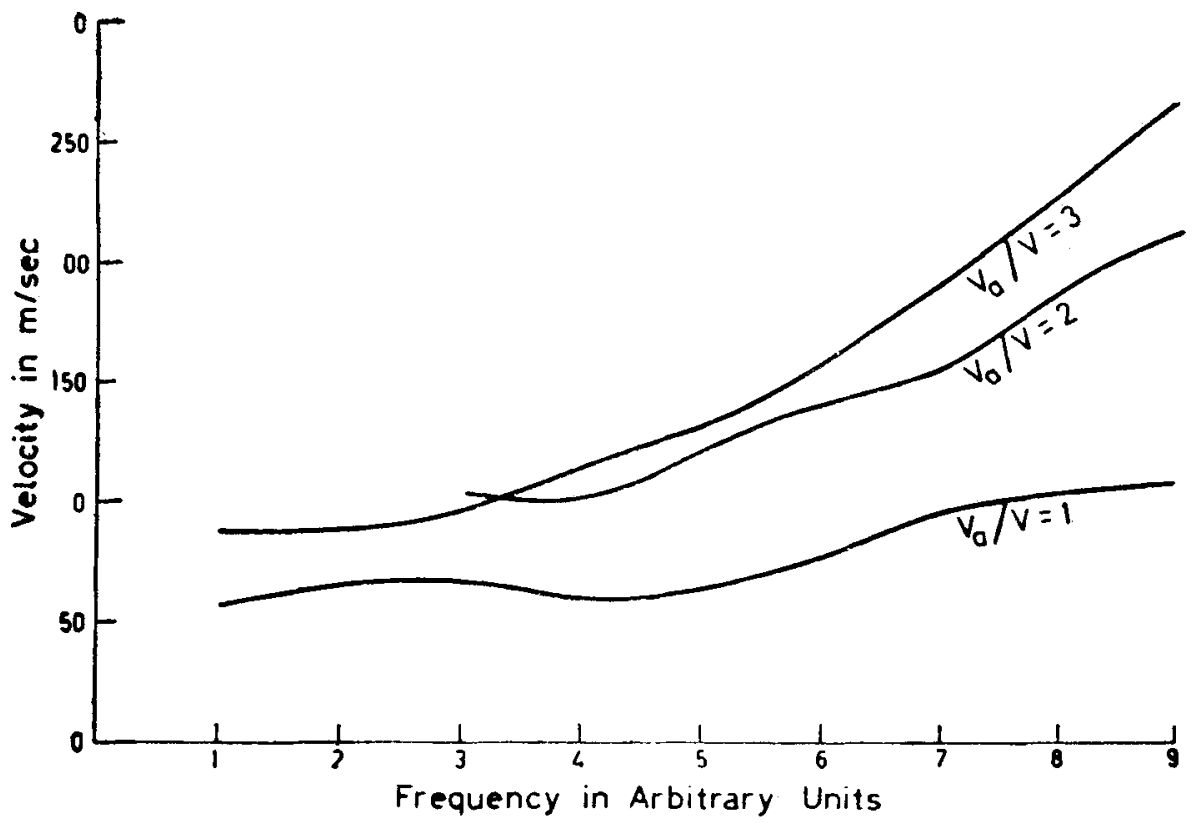

Figure 4. Variation of the drift speed $V_{a}$ with frequency derived from cross-spectral method.

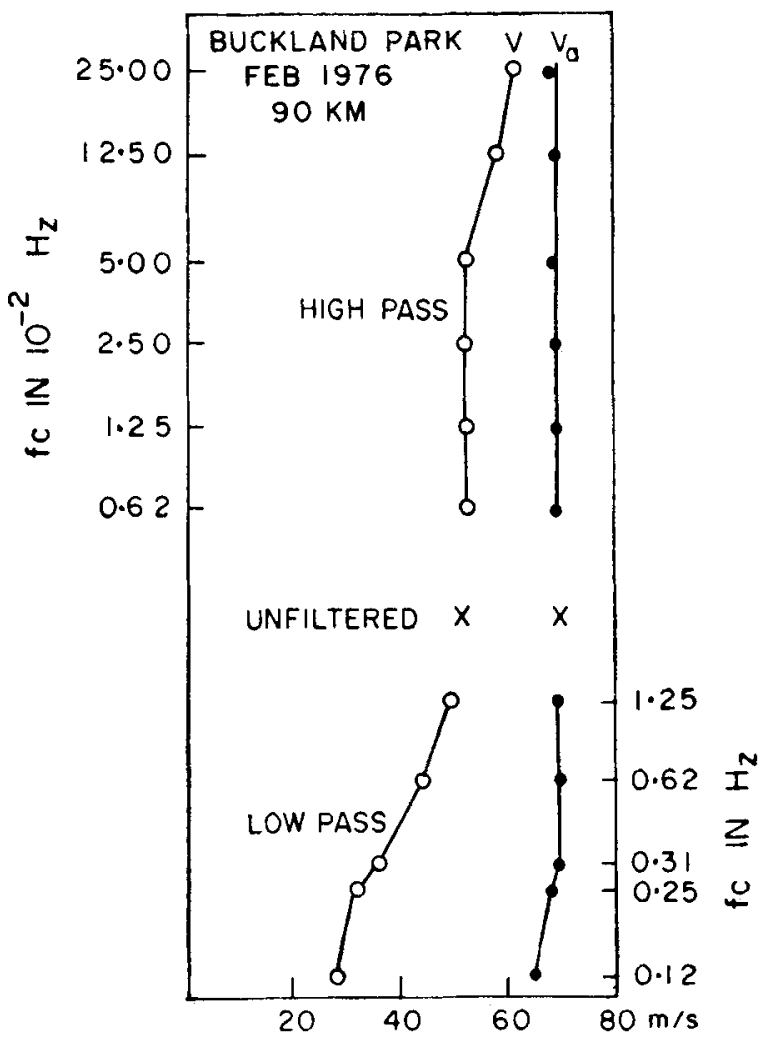

Figure 5. Variation of $V_{a}$ and $V$ with cut-off frequency of low pass and high pass filters. 
subjected to full correlation analysis, which will be free from any end effects due to the use of wide filters.

The two curves in the lower half of figure 5 represent the variations of $V$ and $V_{a}$ with cut-off frequency of the low pass filters while the two curves in the upper half of the figure represent similar variations in case of high pass filters. The two crosses in the middle represent values of $V$ and $V_{a}$ for unfiltered samples.

The apparent drift speed $V_{a}$ remains independent of the cut-off frequency of the high pass filters. The true drift speed $V$ also remains constant initially but with increasing cut-off frequency it starts increasing and comes close to the apparent drift speed.

For the case of low pass filters the true drift speed $V$ decreases as the cut-off frequency of the low pass filter is decreased. The apparent drift speed remains constant initially but when the cut-off frequency is very low shows a small decrease (which is much less ccmpared to the decrease in the true drift speed). Thus while the apparent drift speed remained constant, the true drift speed varied considerably with the filter frequency. We conclude that the dispersion is not present for the large number of records analysed at Buckland Park.

\section{Conclusion}

On account of the results presented here it appears that the true drift velocities derived from full correlation analysis are more correct. Records with large variations with velocity with time can give rise to very high apparent drift velccities which must be rejected by allowing some appropriate pre-conditions in the computer programme. Physical interpretations of dispersion analysis are still not well understood and the variation of velocity with frequency is most likely to be due to the variations in the velocity with time.

\section{Acknowledgements}

The author is grateful to Dr B H Briggs for suggesting this problem. Sincere thanks are also due to Drs $R$ A Vincent and $T J$ Stubbs for help and discussions. The work was supported by the Australian Research Grants Committee.

\section{References}

Briggs B H, Phillips G J and Shinn D H 1950 Proc. Phys. Soc. (London) B63 106

Briggs B H 1977 J. Atmos. Terr. Phys. 391023

Chandra H and Briggs B H $1978 \mathrm{~J}$. Atmos. Terr. Phys, 40541

Crochet M, Tabbagh J and Makiesse N 1977 J. Atmos. Terr. Phys. 39463

Felgate D G $1970 \mathrm{~J}$. Atmos. Terr. Phys. 32241

Felgate D G and Golley M G 1971 Aust. J. Phys. 24397

Felgate D G, Hunter A N, Kingsley S P and Muller H G 1975 Planet Space Sci. 23389

Fooks G F 1965 J. Atmos. Terr. Phys. 27979

Golley M G and Rossitor D E 1971 J. Atmos. Terr. Phys. 33701

Jones D and Maude A D 1965 Nature (London) 206177

Mitra S N 1949 Proc. Inst. Electr. Eng. 96441 
Pitteway M L V, Wright J W and Fedor L S 1971 J. Atmos. Terr. Phys. 33635

Phillips G J and Spencer M 1955 Proc. Phys. Soc. (London) BG\& 481

Sprenger K and Schminder R 1969 J. Atmos. Terr. Phys. 31 1085

Stubbs T J 1973 J. Atmos. Terr. Phys. 38979

Vincent R A, Stubb T J, Pearson P H O, Lloyd K H and Low C H 1977 J. Atmos. Terr. Phys. 39813

Wright J W, Glass M and Spinnichino A 1976a J. Atmos. Terr. Phys. 38713

Wright J W, Amayenc P and vasseur G 1976b J. Atmos, Terr. Phys. 38731 